\title{
Management system of a sustainable organization: attributes and factors
}

\author{
Angelina Dolgaya ${ }^{1, *}$, and Olga Kovbasyuk ${ }^{2}$ \\ ${ }^{1}$ Kaliningrad State Technical University, Kaliningrad, Russia \\ ${ }^{2}$ I. Kant Baltic Federal University, Kaliningrad, Russia
}

\begin{abstract}
The purpose of the study is to identify the main features of the management system of a sustainable organization and the driving forces of its development. The results of the study are used to create a conceptual model of the management system of a sustainable organization. To achieve this goal, the authors analyzed the existing principles, approaches and management models of sustainable organizations both in the form of cases of successful companies and in a generalized form. The analysis was carried out according to the authors' structure of the architectonics of the organization management system. The features of the management system of a sustainable business organization were discovered, among which the key place, as a factor of its development, is the set of values of the leader. This specific set of values is called "sustainability", and it determines the appropriate functioning of other elements of the system, such as organizational culture, performance indicators, and internal standards. The most significant sign of the influence of the set of values "sustainability" on the management system are investment decisions, and in particular, the choice of production technologies in the development of the company. The study confirmed the need for a comprehensive approach to assessing the sustainability of business organizations. The necessity of forming a set of values of leaders and owners of companies as the main factor in the development of sustainable business ecosystems is also revealed.
\end{abstract}

\section{Introduction}

For several decades, humanity has tried to solve the problems of economic inequality through industrialization, increasing consumption of natural resources in the hope that this will help to get rid of poverty. But this path did not lead to universal economic prosperity, it destroyed the balance in the earth's ecosystem, on the contrary [1].

Any sustainable development of a high-order system, such as humanity, a country, and a community, is the result of the interaction of its subsystems. At the same time, subsystems should be focused in their activities precisely on the common goals of sustainable development of a common system for all. In this sense, companies act as subsystems for sustainable development of all mankind on the planet Earth. But how should they function to promote universal sustainability?

\footnotetext{
* Corresponding author: dolgaya@dialoglan.ru
} 
We hold that sustainable development is located at the heart of the company's existence, when sustainability indicates resource consumption reduction on the one hand, and company growth due to increasing customer satisfaction, on the other. However, even with these indicators in action, not every company might be regarded as a sustainable one [2].

Another factor to be considered for a sustainable company, is its capability of concentrating on company present needs without bringing harm to the future needs $[1,3]$. As a consequence, a sustainable organization might be the one, which achieves its current goals without compromising the ability of future long-term development.

To illustrate further considerations for sustainability, we proceed to 17 Sustainable Development Goals, adopted by the UN, three of which directly affect the principles and approaches to companies management [4]. In other words, three goals out of seventeen seem to lead business companies to sustainability:

- Promotion of sustained, inclusive and sustainable economic growth, full and productive employment and decent work for all $\left(8^{\text {th }} \mathrm{Goal}\right)$

- Provision of sustainable consumption and production models $\left(12^{\text {th }} \mathrm{Goal}\right)$

- Building resilient infrastructure, promotion of inclusive and sustainable industrialization and fostering innovation $\left(9^{\text {th }}\right.$ Goal $)$

The purpose of the research is to analyze the latest concepts and findings in understanding the mechanism of sustainable development and to search for the necessary modifications in the architectonics of the company's management system developed by the author [5].

\section{Materials and methods}

The earlier proposed concept of an organization management system called its architectonics, can be represented as a purposeful and unconscious influence of a leader on technology and standards of doing business, represented in a governance model [5]. This very concept serves not only to reflect the fundamental relationships between various elements within the management system, but vectors of influence and determinants of these elements. It also emphasizes the importance of human-centered creativity in the organization management.

Having this concept in mind, we proceed further to Sustainable Development Goals to explore the architectonics of the company's management system in more details. What does it take for it to achieve the goals of humanity sustainable functioning in the ecosystem of our planet? This question opens up a number of areas to study the interaction of control system elements, including:

- how does a company leader influence the way to achieve Sustainable Development Goals?;

- what is the place of the governance model in the management architecture of a sustainable company?;

- what governance mechanisms can determine the achievement of the Sustainable Development Goals?;

- what organizational standards should characterize a sustainable company?

In addition to considering these questions, we need to identify a specific configuration of the management system for a sustainable company, which is important to do in order to achieve the goals set by the UN.

The stated purpose and questions of the study configure the conceptual nature of this article. Systematic literature review is a key research method [6,7] applied to find the results of the corresponding focus.

Also, to modify Dolgaya's model of management architectonics based on the system approach, we used the method of synthesis of conclusions obtained from the analysis of the literature and recent studies. When analyzing the existing concepts and trends in the topic of 
sustainable business development management, the method of abstraction was used to form corresponding hypotheses for architectonic specifications.

\subsection{Shortcoming: Sustainability, as a Goal, is Separated from a Company Business Strategy}

We believe that sustainable competitive advantage in business is not currently associated with sustainability in the global understanding of the prospects for the development of the ecosystem "humanity on planet Earth" [8]. At the same time, each company is at its own level of integration of the principles of sustainability into its management system. The depth of integration of the principles of sustainability in life of a corporation is defined by BCG in the following four levels: corporate social responsibility, compliance-driven orientation, reactive changes and business model innovation. According to the recent research [9], the most profound changes towards sustainability are realized through transformation of a business model. The second level of integration-compliance driven orientation -the focus on sustainability creates the problem of separating business strategy with formal requirements of sustainable development. For example, progress in achieving sustainability goals is not directly related to progress in creating competitive advantages, productivity growth, and financial viability. Operational and annual management at this level is focused on financial returns. Compliance with sustainability goals is considered separately, through the prism of a special set of indicators, often with separate reporting and weak connection to the value chain [9]. BCG points out the need for comprehensive changes in a management system, that affect not only its individual indicators, but also the scale of company's activity, including geography of business, company partnerships, social and environmental trends.

\subsection{Shortcoming: Sustainability is not Integrated into Business Ecosystem}

When building a management model for sustainability purposes, managers focus on the company's reporting. Sometimes individual elements of the supply chain are included in the overall management model, but very rarely the entire business ecosystem, entire industry - all stakeholders that influence the achievement of sustainability are integrated into it [10]. This fragmentation of the overall flow of value creation and consumption of goods or services is a critical constraint to the implementation of sustainable business principles.

\subsection{Opportunities: New Technology for a Sustainable Management Model}

The Fourth Industrial Revolution (4IR) makes it possible to increase productivity without additional consumption of capital resources, but only by increasing efficiency of available resources application. This is especially evident in processing industries [2]. 53\% of lighthouse cases show the obvious impact of 4IR on sustainability. More than $69 \%$ of processing industries report sustainability KPI impact as a result of digitalization, and $8 \%$ report direct impact of KPI on sustainability through process and machine optimization, predictive management and product planning.

Logistics interactions processes between a manufacturer, sales channels and a consumer demonstrate the largest costs reduction due to web-based platforms, as in the case with Johnson\&Johnson. At Bosch, digital shift performance management and a digitally enabled automatic material call-off system, along with cycle time and changeover optimization powered by machine vision, have improved worker and machine capacity while boosting quality and reducing costs. Novo Nordisk has coupled digital scheduling and work- 
management apps with production line optimization, automated OEE monitoring and digital performance management to release capacity.

According to World Economic Forum [2] not only manufacturing processes change the frame of sustainability operations, but governance models and business models serve the purpose. For example, Henkel initial commitment to achieve a $65 \%$ carbon footprint reduction by 2025 at its production sites got materialized eventually in peer network changeover. Benchmarks of paper replacement by software, energy efficiency, product obsolescence elimination were introduced as a criteria for cooperation. This network with more than 30 factories worldwide, demonstrated $70 \%$ reduction of paper production, $35 \%$ waste reduction, and $16 \%$. energy consumption reduction. To further drive improvements in productivity and boost the company's sustainability, Henkel built its digital backbone to scale 4IR technologies linking its cyber and physical systems across its plant in Montornès, Spain, thus reducing costs by $15 \%$ and accelerating time to enter the market by $30 \%$ while shrinking its carbon footprint by $10 \%$, as CEO holds [11].

\subsection{Opportunities: New System Management Solutions}

In the Gartner 2020 Future of Supply Chain Survey, 84\% of Corporate Supply Chain Officers report they plan to invest in climate adaptation and mitigation measures in the next 18 months (Gartner, 2021) [12]. It means sustainability goals turn more and more timely not only in the processing industry, but in business in general. Moreover, a business model for sustainability goals has appeared. It incorporates sustainability as an integral part of the company's value proposition and value creation logic. As such, business models for sustainability goals provide value to a customer and to natural environment and/or society [13].

In order to find out how leading companies are changing their business models, Tis and Boken conducted a series of interviews with top managers of such companies as AkzoNobel, Interface, Johnson \& Johnson, Pearson Education, Philips, UBS and Unilever. They found five important elements to build a business model for a sustainable organization [14].

First, a company must be very clear about the direction of its sustainable development and communicate the relevant goals to all employees. Secondly, sustainability requires the availability of appropriate resources, including time, information, and training, to achieve the goals set. Third, it is necessary to have a "room for collaboration", that is, the ability for people to freely interact within the company, as well as with suppliers and partners as well as with other organizations, in order to satisfy the need for missing competencies or resources. Fourth, company management must provide consistent positive reinforcement for all initiatives aimed at improving organization sustainability. Finally, a business model should include standards for accounting and monitoring the process of creating social and environmental value, as one of the most important priorities. Thus, from this study, we can conclude that the main elements of a business model for a sustainable organization are a governance model (translation of the sustainable development goals throughout the company), performance standards that support sustainability and the appropriate organizational culture.

To further explore the new management solutions, we turn to the general principles of the phenomenon of sustainability in business environment, which have been determined as a result of analysis of a fairly large list of corporate and NGO standards [15]. The nine identified principles of sustainable development are ethics, governance, transparency, business relations, financial return, community participation, product value, employment practices, and environmental care. The authors of this study pointed out the need to not only set the goals for a sustainable strategy, but also the means to measure their implementation. For example, regarding ethics as an introduced principle, Epstein recommends creating a 
code of conduct in a company, that applies not only to its employees, but also to its suppliers, distributors, and other business partners. He defines sustainable relationships as long-term relationships with suppliers in exchange for quality and competitiveness. When interacting with a community, each company must determine a degree of its openness and transparency. One of the values of such interaction, as he holds, is understanding of company's unity with the environment and with other people in this environment.

The authors' findings [16] add to ethics principle value, as they proved that standards, principles, and rules under which an organization operates are created not only by formal management decisions, but also by unwritten norms of behavior, priorities/values of company managers, and the overall strength of organizational culture. It is important that employees at all levels understand that ethical violations are unacceptable and will have irreversible consequences.

\subsection{Opportunities: Sustainability as a Value System}

Robert Dilts defines sustainability as balance between the ego and the soul - between an individualistic perception of oneself and, on the contrary, a perception of oneself as part of a whole, but without losing individuality [17]. The leader of an organization, like any other person, has his/her own model of balancing personal and global values (Fig.1). At the same time, it turns out that at the deepest levels of personality manifestation, the problem of choosing between personal and general, between individual needs and community needs, between the country and the entire ecosystem arises.

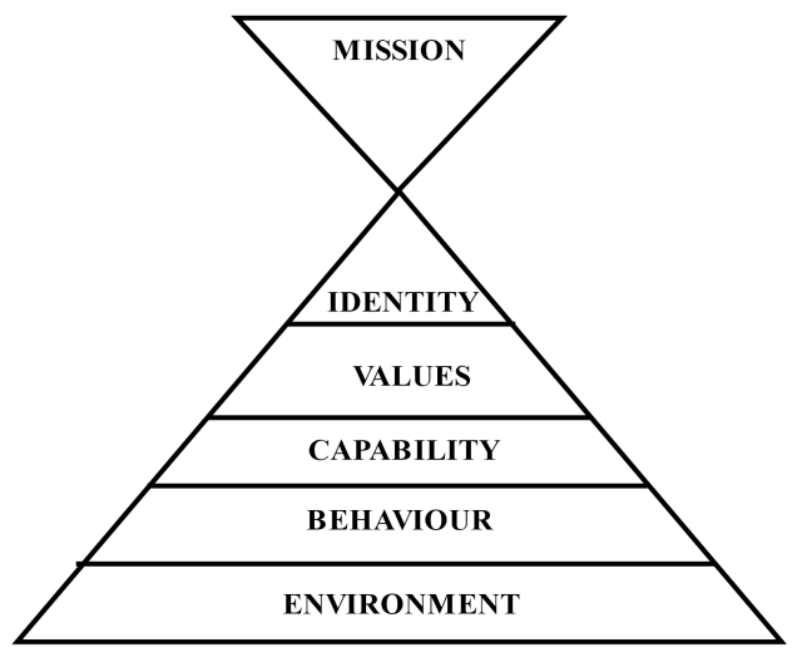

Fig. 1. Neurological levels of development [17].

We agree that the values transmitted by a leader to an organization are determined precisely by the balance of a deeper level - the awareness of their role as a subsystem in a more global system.

Simon Sinek also emphasizes the importance of a business mission as a resolution on the "Why Question" for leaders of a company. The long term strategic success and competitiveness depends very much on the real balance between an individual purpose and more spiritual goals like service to and connection with something bigger than an individual, ability to make contributions to a community [18].

Back in 1995, the World Business Council for Sustainable Development was established, bringing together more than 200 CEOs. This organization initiates a number of actions, 
including a Redefining Value program, which includes the creation of vision 2050, projects for development of Assess and Manage Performance, and a number of projects for external disclosure transparency. However, we believe they place too much hope on the company's board of directors. At the same time, this body itself recognizes that sustainability is very rarely integrated into a business management system. But at the same time, the body aims at working with key stakeholders to include sustainability in a management strategic decisionmaking model. For 6 years, the Redefining Values Program has been responding to a request from top managers to redesign management models for sustainability.

Another strong attribute of sustainability is organizational culture, as we stated earlier. Strong organizational culture means shared values between employees [19]. Our research [16] confirmed the fact of linear dependance between strong organizational culture and leader's ability to build up a desired governance model.

Shared values system is demonstrated by family run business in the form of transgenerational succession. It can also be associated with the adoption of sustainable practices [20]. In this case, the values of business owners are beyond their current needs and concern for short-term profits is not a priority criterion when making management decisions. In doing so, they illustrate and confirm our definition of sustainability as prioritizing current goals over long-term goals that go beyond a single human lifetime.

\section{Results and Discussion}

The problems, opportunities, and underlying factors of sustainable companies that we have identified require systematization. Following the structure of the architectonics of a company management system, we can formulate its main features for a sustainable organization.

Attributes of a sustainable organization management system are:

— balanced set of "Sustainability" priority values;

— integration of "Sustainability" values set into a governance model;

- compliance of corporate norms and standards with "Sustainability" set of values.

The following relationships of these features in management system architectonics (Fig.2) establishes a truly stable design, provided that each of them is present.

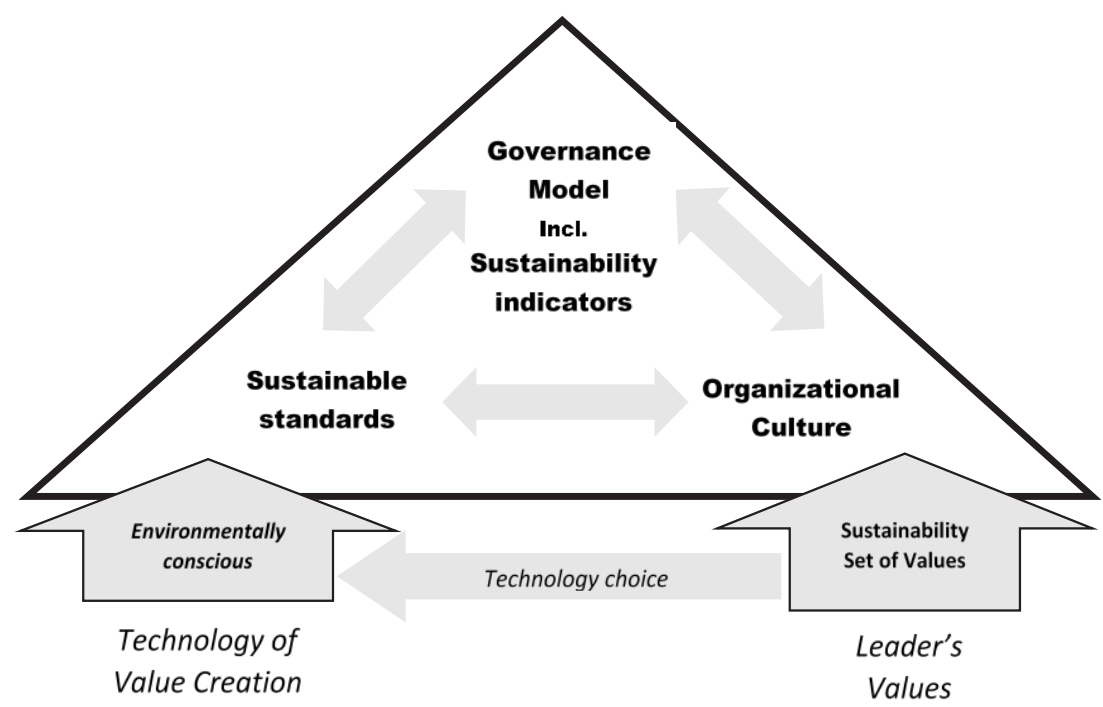

Fig.2. Management system architectonic of sustainable organization. 


\subsection{Balanced Set of "Sustainability" Priority Values}

Companies able of cooperating at the industry level and cooperating with competitors, able of forming communities can be considered sustainable.

We hold that the foundation for a sustainable business model is Simon Seneca's Golden Circle: the mission must be compliant with company's goals and practices. Where company's mission goes beyond Ego level, there is the ability to cooperate to the detriment of individual interests.

\subsection{Integration of "Sustainability" Values Set of into a Governance Model}

The first and fifth Epstein principles confirm the need to create a single sustainable management model. Such a model should include not only values, but also parameters for evaluating results built upon values (Epstein). A sustainable governance model assumes the existence of an appropriate system for evaluating the company's performance, based on a specific system of indicators. These indicators include the well-being indicators [21].

If sustainability is an alien value for a company, then the requirements for achieving it can only be measured by compliance indicators. In this case, the company is only at the second level of integration and cannot be considered truly sustainable. An attribute of the sustainability for a governance model is the congruence of a declared mission of a company with its long-term strategy and with operational performance indicators.

\subsection{Compliance of Corporate Norms and Standards with "Sustainability" Set of Values}

The normal long-term functioning of an industry and any other business communities involves the full adoption of agreed standards of behavior, an agreed level of openness and trust. That is, the condition for the existence of such communities is also a congruence of values and meanings, among which the most important are trust, openness and an established balance between individual and universal interests.

\subsection{Factors of the Management System of a Sustainable Organization}

The sustainability of the business from the point of view of the environment and long-term interests is determined precisely by the correspondence of the value position to the selected technologies for creating consumer value.

Thus, the main factors for creating a management system for a sustainable organization can be considered as:

- a prerequisite for sustainability is the sustainability set of values inherent in the leader of the organization;

- a congruence of values (principles) of leader and key performance indicators of the governance model;

- the production technology selected in accordance with the principles of sustainability

The choice of technology for production processes is a litmus test for the leaders and owners of the company. At the time of selection, it is checked whether the real values coincide with the declared values of sustainability.

The main task of further research is to identify the set of values of "Sustainability". After clarifying the list of these values, it will become possible to hypothesize the interaction of elements of the architectonics of the management system of a sustainable organization. The beginning of such a review should be to assess the priorities of the values of the leader of the organization in comparison with the priorities of the employees. The subsequent management 
system sustainability study will focus on comparing the company's mission and long-term strategy implementation parameters, with operational key performance indicators.

\section{Conclusion}

To summarize all the findings, we hold that a management system of a sustainable organization is characterized by a complex of interrelated features. In accordance with the architecture of the management system, these features should include not only the appropriate set of performance indicators, but also organizational standards that cover the entire partner network of the company, from suppliers to consumers. A prerequisite for sustainability is an adopted sustainability set of values, including:

- Values of a leader, priority of long-term goals over short-term ones;

- Organizational culture, predominance of value priorities that coincide with leader values;

- System of planning, control, and staff incentives, priority of long-term evaluation indicators over short-term ones.

- A choice of production technology, where the production impact on the environment is more important than the price of the equipment.

With all these elements of management system architectonics integrated into one, their corresponding mutual influence will give confidence that the company operates on the principles of sustainability and is able of achieving the goal of sustainable development.

\section{Reference}

1. Our Common Future: Report of the World Commission on Environment and Development, http://www.un-documents. net/

2. Global Lighthouse Network: Reimagining Operations for Growth (2021)

3. The Desirability and Feasibility of ISO Corporate Social Responsibility Standards (2002)

4. Unated Nations, https://sdgs.un.org/

5. A. A Dolgaya, Scientific notes of the Russian academy of entrepreneurship, 38, 44 (2014)

6. D. Tranfield, D. Denyer, P. Smart (2003), British J. of Management, 14(3), 207 (2003)

7. B. A. Kitchenham, S. Charters, Guidelines for performing systematic literature reviews in software engineering version 2.3 (2007)

8. D. Young, M. Reeves, The Quest for Sustainable Business Model Innovation (2020)

9. R. Edgeman, J. A. Williams, The TQM J., 26(4), 368 (2014)

10. G. Roos, Estrategia, 9, 66 (2020)

11. CEO dialogue: Perspectives on reimagining operations for growth (2021)

12. Supply Chain Sustainability Trends (2021)

13. Casadesus-Masanell \& Ricart (2007)

14. T. Geradts, N. Bocken, MIT Sloan Management, 28 (2018)

15. J. M. Epstein, M.-J. Roy, J. of General Management, 29(1) (2003)

16. A. Dolgaya, O. Kovbasyuk, V. Altunina, Organizational Culture Power as a Factor of Governance Model Implementation E3S Web Conf., 20806013 (2020) 
17. R. Dilts, The PERICEO Tool: Teams and Organizations, Develop Your Capacity for Collective Intelligence (2019)

18. S. Sinek, Find Your Why: A Practical Guide for Discovering Purpose for You and Your Team (2017)

19. S. P. Robbins, Organizational Behavior: concepts, controversies, applications (1996)

20. Sustainable Certification for Future Generations: The Case of Family Business Magali A. Delmas and Olivier Gergaud Family Business (2014)

21. M. Delmas, R. Durand, Measuring Business Impacts on Well-Being: A Goal Oriented Approach (2018) 